\title{
Adaptive Controller Design for THE HYBRID SYNCHRONIZATION OF HYPERCHAOTIC XU AND HYPERCHAOTIC Li SYSTEMS
}

\author{
Sundarapandian Vaidyanathan ${ }^{1}$ \\ ${ }^{1}$ Research and Development Centre, Vel Tech Dr. RR \& Dr. SR Technical University \\ Avadi, Chennai-600 062, Tamil Nadu, INDIA \\ sundarvtuegmail.com
}

\begin{abstract}
This paper derives new adaptive results for the hybrid synchronization of hyperchaotic Xi systems (2009) and hyperchaotic Li systems (2005). In the hybrid synchronization design of master and slave systems, one part of the systems, viz. their odd states, are completely synchronized (CS), while the other part, viz. their even states, are completely anti-synchronized (AS) so that CS and AS co-exist in the process of synchronization. The research problem gets even more complicated, when the parameters of the hyperchaotic systems are unknown and we tackle this problem using adaptive control. The main results of this research work are proved using adaptive control theory and Lyapunov stability theory. MATLAB simulations using classical fourth-order Runge-Kutta method are shown for the new adaptive hybrid synchronization results for the hyperchaotic Xu and hyperchaotic Li systems.
\end{abstract}

\section{KEYWORDS}

Hybrid Synchronization, Adaptive Control, Chaos, Hyperchaos, Hyperchaotic Systems.

\section{INTRODUCTION}

The hyperchaotic system was first discovered by the German scientist, O.E. Rössler ([1], 1979). It is a nonlinear chaotic system having two or more positive Lyapunov exponents. Hyperchaotic systems have many attractive features and hence they are applicable in areas like neural networks [2], oscillators [3], communication [4-5], encryption [6], synchronization [7], etc.

For the synchronization of chaotic systems, there are many methods available in the chaos literature like OGY method [8], PC method [9], backstepping method [10-12], sliding control method [13-15], active control method [16-17], adaptive control method [18-19], sampled-data feedback control [20], time-delay feedback method [21], etc.

In the hybrid synchronization of a pair of chaotic systems called the master and slave systems, one part of the systems, viz. the odd states, are completely synchronized (CS), while the other part of the systems, viz. the even states, are anti-synchronized so that CS and AS co-exist in the process of synchronization of the two systems.

This paper focuses upon adaptive controller design for the hybrid synchronization of hyperchaotic Xu systems ([22], 2009) and hyperchaotic Li systems ([23], 2005) with unknown parameters. The

DOI : $10.5121 /$ ijait.2013.3202 
main results derived in this paper have been proved using adaptive control theory [24] and Lyapunov stability theory [25].

\section{Adaptive Control Methodology fOr Hybrid SynChronization}

The master system is described by the chaotic dynamics

$$
\dot{x}=A x+f(x)
$$

where $A$ is the $n \times n$ matrix of the system parameters and $f: R^{n} \rightarrow R^{n}$ is the nonlinear part. The slave system is described by the chaotic dynamics

$$
\dot{y}=B y+g(y)+u
$$

where $B$ is the $n \times n$ matrix of the system parameters and $g: R^{n} \rightarrow R^{n}$ is the nonlinear part For the pair of chaotic systems (1) and (2), the hybrid synchronization error is defined as

$$
e_{i}=\left\{\begin{array}{cc}
y_{i}-x_{i}, & \text { if } i \text { is odd } \\
y_{i}+x_{i}, & \text { if } i \text { is even }
\end{array}\right.
$$

The error dynamics is obtained as

$$
\dot{e}_{i}= \begin{cases}\sum_{j=1}^{n}\left(b_{i j} y_{j}-a_{i j} x_{j}\right)+g_{i}(y)-f_{i}(x)+u_{i} & \text { if } i \text { is odd } \\ \sum_{j=1}^{n}\left(b_{i j} y_{j}+a_{i j} x_{j}\right)+g_{i}(y)+f_{i}(x)+u_{i} & \text { if } i \text { is even }\end{cases}
$$

The design goal is to find a feedback controller $u$ so that

$$
\lim _{t \rightarrow \infty}\|e(t)\|=0 \text { for all } e(0) \in R^{n}
$$

Using the matrix method, we consider a candidate Lyapunov function

$$
V(e)=e^{T} P e,
$$

where $P$ is a positive definite matrix. It is noted that $V: R^{n} \rightarrow R$ is a positive definite function. If we find a feedback controller $u$ so that

$$
\dot{V}(e)=-e^{T} Q e,
$$

where $Q$ is a positive definite matrix, then $\dot{V}: R^{n} \rightarrow R$ is a negative definite function.

Thus, by Lyapunov stability theory [25], the error dynamics (4) is globally exponentially stable. Hence, the states of the chaotic systems (1) and (2) will be globally and exponentially hybrid synchronized for all initial conditions $x(0), y(0) \in R^{n}$. When the system parameters are unknown, we use estimates for them and find a parameter update law using Lyapunov approach. 


\section{HYPERCHAOTIC SYSTEMS}

The hyperchaotic Xu system ([22], 2009) has the 4-D dynamics

$$
\begin{aligned}
& \dot{x}_{1}=a\left(x_{2}-x_{1}\right)+x_{4} \\
& \dot{x}_{2}=b x_{1}+r x_{1} x_{3} \\
& \dot{x}_{3}=-c x_{3}-x_{1} x_{2} \\
& \dot{x}_{4}=x_{1} x_{3}-d x_{2}
\end{aligned}
$$

where $a, b, c, r, d$ are constant, positive parameters of the system.

The Xu system (8) exhibits a hyperchaotic attractor for the parametric values

$$
a=10, \quad b=40, \quad c=2.5, \quad r=16, \quad d=2
$$

The Lyapunov exponents of the system (8) for the parametric values in (9) are

$$
\lambda_{1}=1.0088, \quad \lambda_{2}=0.1063, \quad \lambda_{3}=0, \quad \lambda_{4}=-13.6191
$$

Since there are two positive Lyapunov exponents in (10), the Xu system (8) is hyperchaotic for the parametric values (9).

The strange attractor of the hyperchaotic Xu system is displayed in Figure 1.

The hyperchaotic Li system ([23], 2005) has the 4-D dynamics

$$
\begin{aligned}
& \dot{x}_{1}=\alpha\left(x_{2}-x_{1}\right)+x_{4} \\
& \dot{x}_{2}=\delta x_{1}-x_{1} x_{3}+\gamma x_{2} \\
& \dot{x}_{3}=-\beta x_{3}+x_{1} x_{2} \\
& \dot{x}_{4}=x_{2} x_{3}+\varepsilon x_{4}
\end{aligned}
$$

where $\alpha, \beta, \gamma, \delta, \varepsilon$ are constant, positive parameters of the system.

The Li system (11) exhibits a hyperchaotic attractor for the parametric values

$$
\alpha=35, \quad \beta=3, \quad \gamma=12, \quad \delta=7, \quad \varepsilon=0.58
$$

The Lyapunov exponents of the system (11) for the parametric values in (12) are

$$
\lambda_{1}=0.5011, \quad \lambda_{2}=0.1858, \quad \lambda_{3}=0, \quad \lambda_{4}=-26.1010
$$

Since there are two positive Lyapunov exponents in (13), the Li system (11) is hyperchaotic for the parametric values (12).

The strange attractor of the hyperchaotic Li system is displayed in Figure 2. 

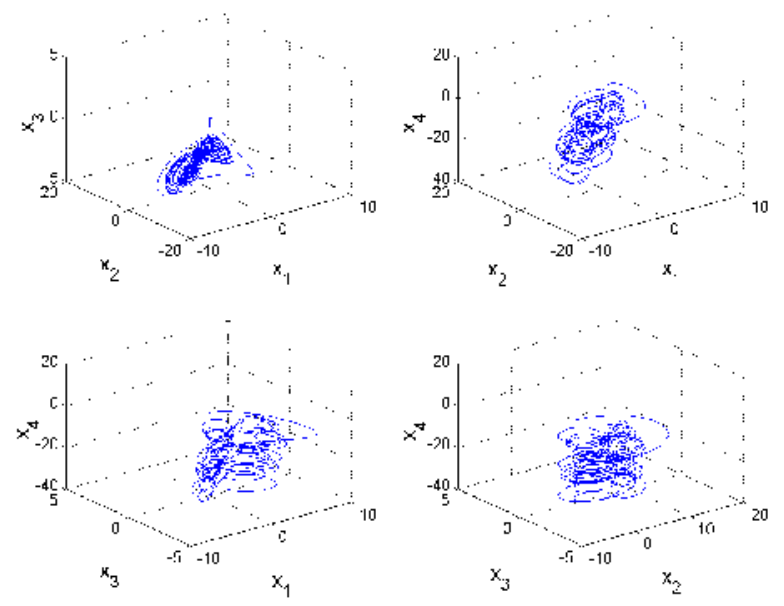

Figure 1. The State Portrait of the Hyperchaotic Xu System
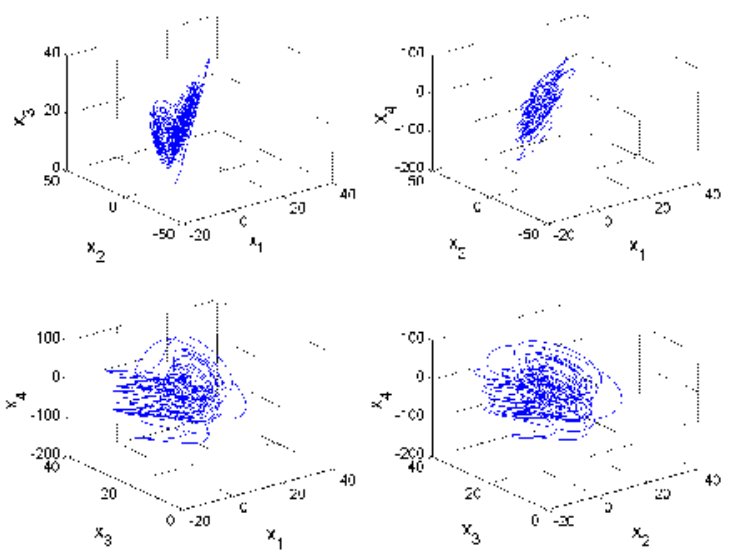

Figure 2. The State Portrait of the Hyperchaotic Li System

\section{Adaptive Control Design for the Hybrid Synchronization of HYPERCHAOTIC XU SYSTEMS}

In this section, we design an adaptive controller for the hybrid synchronization of two identical hyperchaotic Xu systems (2009) with unknown parameters.

The hyperchaotic $\mathrm{Xu}$ system is taken as the master system, whose dynamics is given by

$$
\begin{aligned}
& \dot{x}_{1}=a\left(x_{2}-x_{1}\right)+x_{4} \\
& \dot{x}_{2}=b x_{1}+r x_{1} x_{3} \\
& \dot{x}_{3}=-c x_{3}-x_{1} x_{2} \\
& \dot{x}_{4}=x_{1} x_{3}-d x_{2}
\end{aligned}
$$

where $a, b, c, d, r$ are unknown parameters of the system and $x \in R^{4}$ is the state of the system. 
The hyperchaotic Xu system is also taken as the slave system, whose dynamics is given by

$$
\begin{aligned}
& \dot{y}_{1}=a\left(y_{2}-y_{1}\right)+y_{4}+u_{1} \\
& \dot{y}_{2}=b y_{1}+r y_{1} y_{3}+u_{2} \\
& \dot{y}_{3}=-c y_{3}-y_{1} y_{2}+u_{3} \\
& \dot{y}_{4}=y_{1} y_{3}-d y_{2}+u_{4}
\end{aligned}
$$

where $y \in R^{4}$ is the state and $u_{1}, u_{2}, u_{3}, u_{4}$ are the adaptive controllers to be designed using estimates $\hat{a}(t), \hat{b}(t), \hat{c}(t), \hat{d}(t), \hat{r}(t)$ of the unknown parameters $a, b, c, d, r$, respectively.

For the hybrid synchronization, the error $e$ is defined as

$$
e_{1}=y_{1}-x_{1}, e_{2}=y_{2}+x_{2}, e_{3}=y_{3}-x_{3}, e_{4}=y_{4}+x_{4}
$$

A simple calculation gives the error dynamics

$$
\begin{aligned}
& \dot{e}_{1}=a\left(y_{2}-x_{2}-e_{1}\right)+e_{4}-2 x_{4}+u_{1} \\
& \dot{e}_{2}=b\left(y_{1}+x_{1}\right)+r\left(y_{1} y_{3}+x_{1} x_{3}\right)+u_{2} \\
& \dot{e}_{3}=-c e_{3}-y_{1} y_{2}+x_{1} x_{2}+u_{3} \\
& \dot{e}_{4}=-d e_{2}+y_{1} y_{3}+x_{1} x_{3}+u_{4}
\end{aligned}
$$

Next, we choose a nonlinear controller for achieving hybrid synchronization as

$$
\begin{aligned}
& u_{1}=-\hat{a}(t)\left(y_{2}-x_{2}-e_{1}\right)-e_{4}+2 x_{4}-k_{1} e_{1} \\
& u_{2}=-\hat{b}(t)\left(y_{1}+x_{1}\right)-\hat{r}(t)\left(y_{1} y_{3}+x_{1} x_{3}\right)-k_{2} e_{2} \\
& u_{3}=\hat{c}(t) e_{3}+y_{1} y_{2}-x_{1} x_{2}-k_{3} e_{3} \\
& u_{4}=\hat{d}(t) e_{2}-y_{1} y_{3}-x_{1} x_{3}-k_{4} e_{4}
\end{aligned}
$$

In Eq. (18), $k_{i},(i=1,2,3,4)$ are positive gains and $\hat{a}(t), \hat{b}(t), \hat{c}(t), \hat{d}(t), \hat{r}(t)$ are estimates of the unknown parameters $a, b, c, d, r$, respectively.

By the substitution of (18) into (17), the error dynamics is simplified as

$$
\begin{aligned}
& \dot{e}_{1}=(a-\hat{a}(t))\left(y_{2}-x_{2}-e_{1}\right)-k_{1} e_{1} \\
& \dot{e}_{2}=(b-\hat{b}(t))\left(y_{1}+x_{1}\right)+(r-\hat{r}(t))\left(y_{1} y_{3}+x_{1} x_{3}\right)-k_{2} e_{2} \\
& \dot{e}_{3}=-(c-\hat{c}(t)) e_{3}-k_{3} e_{3} \\
& \dot{e}_{4}=-(d-\hat{d}(t)) e_{2}-k_{4} e_{4}
\end{aligned}
$$

Next, we define the parameter estimation errors as

$$
e_{a}(t)=a-\hat{a}(t), e_{b}(t)=b-\hat{b}(t), e_{c}(t)=c-\hat{c}(t), e_{d}(t)=d-\hat{d}(t), e_{r}(t)=r-\hat{r}(t)
$$


Differentiating (20) with respect to $t$, we get

$$
\dot{e}_{a}(t)=-\dot{\hat{a}}(t), \dot{e}_{b}(t)=-\dot{\hat{b}}(t), \dot{e}_{c}(t)=-\dot{\hat{c}}(t), \dot{e}_{d}(t)=-\dot{\hat{d}}(t), \quad \dot{e}_{r}(t)=-\dot{\hat{r}}(t)
$$

In view of (20), we can simplify the error dynamics (19) as

$$
\begin{aligned}
& \dot{e}_{1}=e_{a}\left(y_{2}-x_{2}-e_{1}\right)-k_{1} e_{1} \\
& \dot{e}_{2}=e_{b}\left(y_{1}+x_{1}\right)+e_{r}\left(y_{1} y_{3}+x_{1} x_{3}\right)-k_{2} e_{2} \\
& \dot{e}_{3}=-e_{c} e_{3}-k_{3} e_{3} \\
& \dot{e}_{4}=-e_{d} e_{2}-k_{4} e_{4}
\end{aligned}
$$

We take the quadratic Lyapunov function

$$
V=\frac{1}{2}\left(e_{1}^{2}+e_{2}^{2}+e_{3}^{2}+e_{4}^{2}+e_{a}^{2}+e_{b}^{2}+e_{c}^{2}+e_{d}^{2}+e_{r}^{2}\right)
$$

which is a positive definite function on $R^{9}$.

When we differentiate (22) along the trajectories of (19) and (21), we get

$$
\begin{aligned}
\dot{V}= & -k_{1} e_{1}^{2}-k_{2} e_{2}^{2}-k_{3} e_{3}^{2}-k_{4} e_{4}^{2}+e_{a}\left[e_{1}\left(y_{2}-x_{2}-e_{1}\right)-\dot{\hat{a}}\right]+e_{b}\left[e_{2}\left(y_{1}+x_{1}\right)-\dot{\hat{b}}\right] \\
& +e_{c}\left[-e_{3}^{2}-\dot{\hat{c}}\right]+e_{d}\left[-e_{2} e_{4}-\dot{\hat{d}}\right]+e_{r}\left[e_{2}\left(y_{1} y_{3}+x_{1} x_{3}\right)-\dot{\hat{r}}\right]
\end{aligned}
$$

In view of Eq. (24), we take the parameter update law as

$$
\begin{array}{lll}
\dot{\hat{a}}=e_{1}\left(y_{2}-x_{2}-e_{1}\right)+k_{5} e_{a}, & \dot{\hat{b}}=e_{2}\left(y_{1}+x_{1}\right)+k_{6} e_{b}, \quad \dot{\hat{c}}=-e_{3}^{2}+k_{7} e_{c} \\
\dot{\hat{d}}=-e_{2} e_{4}+k_{8} e_{d}, & \dot{\hat{r}}=e_{2}\left(y_{1} y_{3}+x_{1} x_{3}\right)+k_{9} e_{r}
\end{array}
$$

Theorem 4.1 The adaptive control law (18) along with the parameter update law (25), where $k_{i},(i=1,2, \ldots, 9)$ are positive gains, achieves global and exponential hybrid synchronization of the identical hyperchaotic Xu systems (14) and (15), where $\hat{a}(t), \hat{b}(t), \hat{c}(t), \hat{d}(t), \hat{r}(t)$ are estimates of the unknown parameters $a, b, c, d, r$, respectively. Moreover, all the parameter estimation errors converge to zero exponentially for all initial conditions.

Proof. We prove the above result using Lyapunov stability theory [25].

Substituting the parameter update law (25) into (24), we get

$$
\dot{V}=-k_{1} e_{1}^{2}-k_{2} e_{2}^{2}-k_{3} e_{3}^{2}-k_{4} e_{4}^{2}-k_{5} e_{a}^{2}-k_{6} e_{b}^{2}-k_{7} e_{c}^{2}-k_{8} e_{d}^{2}-k_{9} e_{r}^{2}
$$

which is a negative definite function on $R^{9}$.

This shows that the hybrid synchronization errors $e_{1}(t), e_{2}(t), e_{3}(t), e_{4}(t)$ and the parameter estimation errors $e_{a}(t), e_{b}(t), e_{c}(t), e_{d}(t), e_{r}(t)$ are globally exponentially stable for all initial conditions. 
This completes the proof.

Next, we demonstrate our hybrid synchronization results with MATLAB simulations.

The classical fourth order Runge-Kutta method with time-step $h=10^{-8}$ has been applied to solve the hyperchaotic Xu systems (14) and (15) with the adaptive nonlinear controller (18) and the parameter update law (25). The feedback gains are taken as $k_{i}=5,(i=1,2, \ldots, 9)$.

The parameters of the hyperchaotic Xu systems are taken as in the hyperchaotic case, i.e.

$$
a=10, \quad b=40, \quad c=2.5, \quad r=16, \quad d=2
$$

For simulations, the initial conditions of the hyperchaotic Xu system (14) are chosen as

$$
x_{1}(0)=11, \quad x_{2}(0)=7, \quad x_{3}(0)=9, \quad x_{4}(0)=-5
$$

Also, the initial conditions of the hyperchaotic Xu system (15) are chosen as

$$
y_{1}(0)=3, \quad y_{2}(0)=4, \quad y_{3}(0)=-6, \quad y_{4}(0)=12
$$

Also, the initial conditions of the parameter estimates are chosen as

$$
\hat{a}(0)=4, \hat{b}(0)=11, \quad \hat{c}(0)=-2, \quad \hat{d}(0)=-5, \quad \hat{r}(0)=16
$$

Figure 3 depicts the hybrid synchronization of the identical hyperchaotic Xu systems.

Figure 4 depicts the time-history of the hybrid synchronization errors $e_{1}, e_{2}, e_{3}, e_{4}$.

Figure 5 depicts the time-history of the parameter estimation errors $e_{a}, e_{b}, e_{c}, e_{d}, e_{r}$.
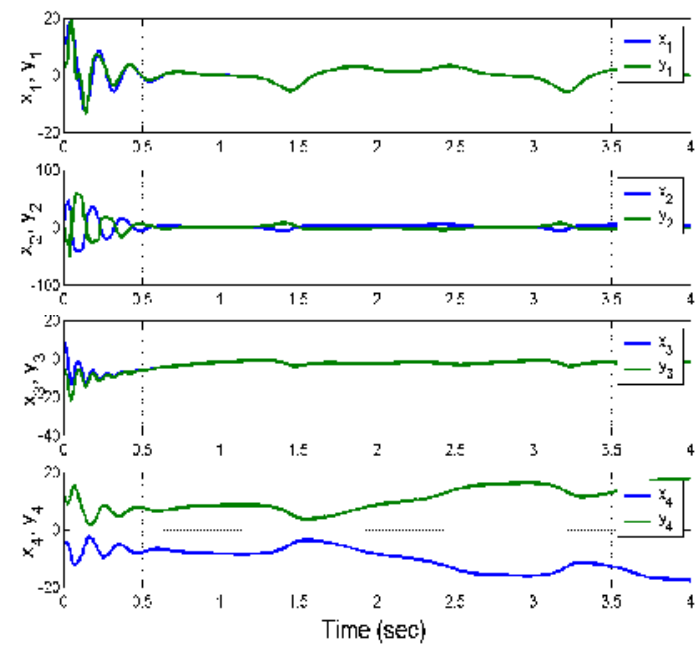

Figure 3. Hybrid Synchronization of Identical Hyperchaotic Xu Systems 


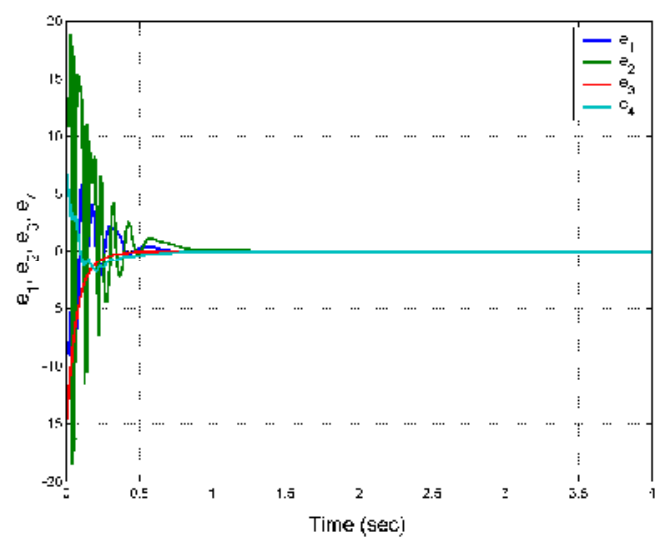

Figure 4. Time-History of the Hybrid Synchronization Errors $e_{1}, e_{2}, e_{3}, e_{4}$

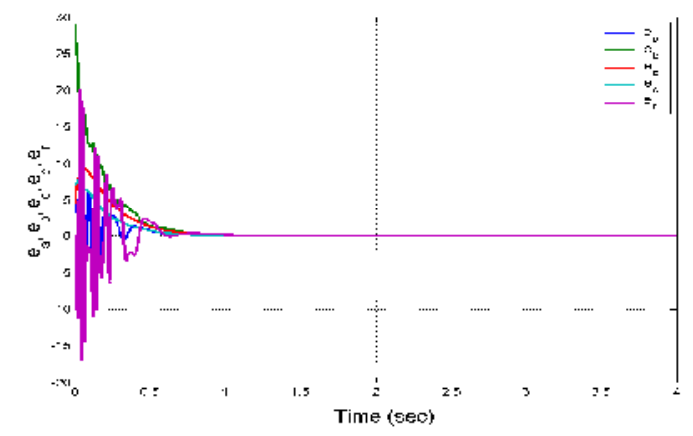

Figure 5. Time-History of the Parameter Estimation Errors $e_{a}, e_{b}, e_{c}, e_{d}, e_{r}$

\section{AdaPtive Controller Design for the Hybrid Synchronization DESIGN OF HYPERCHAOTIC LI SYSTEMS}

In this section, we design an adaptive controller for the hybrid synchronization of two identical hyperchaotic Li systems (2005) with unknown parameters.

The hyperchaotic Li system is taken as the master system, whose dynamics is given by

$$
\begin{aligned}
& \dot{x}_{1}=\alpha\left(x_{2}-x_{1}\right)+x_{4} \\
& \dot{x}_{2}=\delta x_{1}-x_{1} x_{3}+\gamma x_{2} \\
& \dot{x}_{3}=-\beta x_{3}+x_{1} x_{2} \\
& \dot{x}_{4}=x_{2} x_{3}+\varepsilon x_{4}
\end{aligned}
$$

where $\alpha, \beta, \gamma, \delta, \varepsilon$ are unknown parameters of the system and $x \in R^{4}$ is the state of the system. The hyperchaotic Li system is also taken as the slave system, whose dynamics is given by 


$$
\begin{aligned}
& \dot{y}_{1}=\alpha\left(y_{2}-y_{1}\right)+y_{4}+u_{1} \\
& \dot{y}_{2}=\delta y_{1}-y_{1} y_{3}+\gamma y_{2}+u_{2} \\
& \dot{y}_{3}=-\beta y_{3}+y_{1} y_{2}+u_{3} \\
& \dot{y}_{4}=y_{2} y_{3}+\varepsilon y_{4}+u_{4}
\end{aligned}
$$

where $y \in R^{4}$ is the state and $u_{1}, u_{2}, u_{3}, u_{4}$ are the adaptive controllers to be designed using estimates $\hat{\alpha}(t), \hat{\beta}(t), \hat{\gamma}(t), \hat{\delta}(t), \hat{\varepsilon}(t)$ of the unknown parameters $\alpha, \beta, \gamma, \delta, \varepsilon$, respectively.

For the hybrid synchronization, the error $e$ is defined as

$$
e_{1}=y_{1}-x_{1}, e_{2}=y_{2}+x_{2}, e_{3}=y_{3}-x_{3}, e_{4}=y_{4}+x_{4}
$$

A simple calculation gives the error dynamics

$$
\begin{aligned}
& \dot{e}_{1}=\alpha\left(y_{2}-x_{2}-e_{1}\right)+e_{4}-2 x_{4}+u_{1} \\
& \dot{e}_{2}=\delta\left(y_{1}+x_{1}\right)+\gamma e_{2}-y_{1} y_{3}-x_{1} x_{3}+u_{2} \\
& \dot{e}_{3}=-\beta e_{3}+y_{1} y_{2}-x_{1} x_{2}+u_{3} \\
& \dot{e}_{4}=\varepsilon e_{4}+y_{2} y_{3}+x_{2} x_{3}+u_{4}
\end{aligned}
$$

Next, we choose a nonlinear controller for achieving hybrid synchronization as

$$
\begin{aligned}
& u_{1}=-\hat{\alpha}(t)\left(y_{2}-x_{2}-e_{1}\right)-e_{4}+2 x_{4}-k_{1} e_{1} \\
& u_{2}=-\hat{\delta}(t)\left(y_{1}+x_{1}\right)-\hat{\gamma}(t) e_{2}+y_{1} y_{3}+x_{1} x_{3}-k_{2} e_{2} \\
& u_{3}=\hat{\beta}(t) e_{3}-y_{1} y_{2}+x_{1} x_{2}-k_{3} e_{3} \\
& u_{4}=-\hat{\varepsilon}(t) e_{4}-y_{2} y_{3}-x_{2} x_{3}-k_{4} e_{4}
\end{aligned}
$$

In Eq. (31), $k_{i},(i=1,2,3,4)$ are positive gains.

By the substitution of (31) into (30), the error dynamics is simplified as

$$
\begin{aligned}
& \dot{e}_{1}=(\alpha-\hat{\alpha}(t))\left(y_{2}-x_{2}-e_{1}\right)-k_{1} e_{1} \\
& \dot{e}_{2}=(\delta-\hat{\delta}(t))\left(y_{1}+x_{1}\right)+(\gamma-\hat{\gamma}(t)) e_{2}-k_{2} e_{2} \\
& \dot{e}_{3}=-(\beta-\hat{\beta}(t)) e_{3}-k_{3} e_{3} \\
& \dot{e}_{4}=(\varepsilon-\hat{\varepsilon}(t)) e_{4}-k_{4} e_{4}
\end{aligned}
$$

Next, we define the parameter estimation errors as

$$
\begin{aligned}
& e_{\alpha}(t)=\alpha-\hat{\alpha}(t), e_{\beta}(t)=\beta-\hat{\beta}(t), e_{\gamma}(t)=\gamma-\hat{\gamma}(t) \\
& e_{\delta}(t)=\delta-\hat{\delta}(t), e_{\varepsilon}(t)=\varepsilon-\hat{\varepsilon}(t)
\end{aligned}
$$

Differentiating (33) with respect to $t$, we get 


$$
\dot{e}_{\alpha}(t)=-\dot{\hat{\alpha}}(t), \dot{e}_{\beta}(t)=-\dot{\hat{\beta}}(t), \dot{e}_{\gamma}(t)=-\dot{\hat{\gamma}}(t), \dot{e}_{\delta}(t)=-\dot{\hat{\delta}}(t), \quad \dot{e}_{\varepsilon}(t)=-\dot{\hat{\varepsilon}}(t)
$$

In view of (33), we can simplify the error dynamics (32) as

$$
\begin{aligned}
& \dot{e}_{1}=e_{\alpha}\left(y_{2}-x_{2}-e_{1}\right)-k_{1} e_{1} \\
& \dot{e}_{2}=e_{\delta}\left(y_{1}+x_{1}\right)+e_{\gamma} e_{2}-k_{2} e_{2} \\
& \dot{e}_{3}=-e_{\beta} e_{3}-k_{3} e_{3} \\
& \dot{e}_{4}=e_{\varepsilon} e_{4}-k_{4} e_{4}
\end{aligned}
$$

We take the quadratic Lyapunov function

$$
V=\frac{1}{2}\left(e_{1}^{2}+e_{2}^{2}+e_{3}^{2}+e_{4}^{2}+e_{\alpha}^{2}+e_{\beta}^{2}+e_{\gamma}^{2}+e_{\delta}^{2}+e_{\varepsilon}^{2}\right),
$$

which is a positive definite function on $R^{9}$.

When we differentiate (35) along the trajectories of (32) and (33), we get

$$
\begin{aligned}
\dot{V}= & -k_{1} e_{1}^{2}-k_{2} e_{2}^{2}-k_{3} e_{3}^{2}-k_{4} e_{4}^{2}+e_{\alpha}\left[e_{1}\left(y_{2}-x_{2}-e_{1}\right)-\dot{\hat{\alpha}}\right]+e_{\beta}\left[-e_{3}^{2}-\dot{\hat{\beta}}\right] \\
& +e_{\gamma}\left[e_{2}^{2}-\dot{\hat{\gamma}}\right]+e_{\delta}\left[e_{2}\left(y_{1}+x_{1}\right)-\dot{\hat{\delta}}\right]+e_{\varepsilon}\left[e_{4}^{2}-\dot{\hat{\varepsilon}}\right]
\end{aligned}
$$

In view of Eq. (37), we take the parameter update law as

$$
\begin{array}{lll}
\dot{\hat{\alpha}}=e_{1}\left(y_{2}-x_{2}-e_{1}\right)+k_{5} e_{\alpha}, & \dot{\hat{\beta}}=-e_{3}^{2}+k_{6} e_{\beta}, & \dot{\hat{\gamma}}=e_{2}^{2}+k_{7} e_{\gamma} \\
\dot{\hat{\delta}}=e_{2}\left(y_{1}+x_{1}\right)+k_{8} e_{\delta}, & \dot{\hat{\varepsilon}}=e_{4}^{2}+k_{9} e_{\varepsilon}
\end{array}
$$

Theorem 5.1 The adaptive control law (31) along with the parameter update law (38), where $k_{i},(i=1,2, \ldots, 9)$ are positive gains, achieves global and exponential hybrid synchronization of the identical hyperchaotic $\mathrm{Li}$ systems (27) and (28), where $\hat{\alpha}(t), \hat{\beta}(t), \hat{\gamma}(t), \hat{\delta}(t), \hat{\varepsilon}(t)$ are estimates of the unknown parameters $\alpha, \beta, \gamma, \delta, \varepsilon$, respectively. Moreover, all the parameter estimation errors converge to zero exponentially for all initial conditions.

Proof. We prove the above result using Lyapunov stability theory [25].

Substituting the parameter update law (38) into (37), we get

$$
\dot{V}=-k_{1} e_{1}^{2}-k_{2} e_{2}^{2}-k_{3} e_{3}^{2}-k_{4} e_{4}^{2}-k_{5} e_{\alpha}^{2}-k_{6} e_{\beta}^{2}-k_{7} e_{\gamma}^{2}-k_{8} e_{\delta}^{2}-k_{9} e_{\varepsilon}^{2}
$$

which is a negative definite function on $R^{9}$.

This shows that the hybrid synchronization errors $e_{1}(t), e_{2}(t), e_{3}(t), e_{4}(t)$ and the parameter estimation errors $e_{\alpha}(t), e_{\beta}(t), e_{\gamma}(t), e_{\delta}(t), e_{\varepsilon}(t)$ are globally exponentially stable for all initial conditions. This completes the proof. 
International Journal of Advanced Information Technology (IJAIT) Vol. 3, No.2, April2013

Next, we demonstrate our hybrid synchronization results with MATLAB simulations.

The classical fourth order Runge-Kutta method with time-step $h=10^{-8}$ has been applied to solve the hyperchaotic Li systems (27) and (28) with the adaptive nonlinear controller (31) and the parameter update law (38). The feedback gains are taken as $k_{i}=5,(i=1,2, \ldots, 9)$.

The parameters of the hyperchaotic Li systems are taken as in the hyperchaotic case, i.e.

$$
\alpha=35, \quad \beta=3, \quad \gamma=12, \quad \delta=7, \quad \varepsilon=0.58
$$

For simulations, the initial conditions of the hyperchaotic Li system (27) are chosen as

$$
x_{1}(0)=6, \quad x_{2}(0)=-7, \quad x_{3}(0)=15, \quad x_{4}(0)=-22
$$

Also, the initial conditions of the hyperchaotic Li system (28) are chosen as

$$
y_{1}(0)=12, \quad y_{2}(0)=4, \quad y_{3}(0)=9, \quad y_{4}(0)=-6
$$

Also, the initial conditions of the parameter estimates are chosen as

$$
\hat{\alpha}(0)=7, \quad \hat{\beta}(0)=8, \quad \hat{\gamma}(0)=-2, \quad \hat{\delta}(0)=-9, \quad \hat{\varepsilon}(0)=12
$$

Figure 6 depicts the hybrid synchronization of the identical hyperchaotic Li systems.

Figure 7 depicts the time-history of the hybrid synchronization errors $e_{1}, e_{2}, e_{3}, e_{4}$.

Figure 8 depicts the time-history of the parameter estimation errors $e_{\alpha}, e_{\beta}, e_{\gamma}, e_{\delta}, e_{\varepsilon}$.
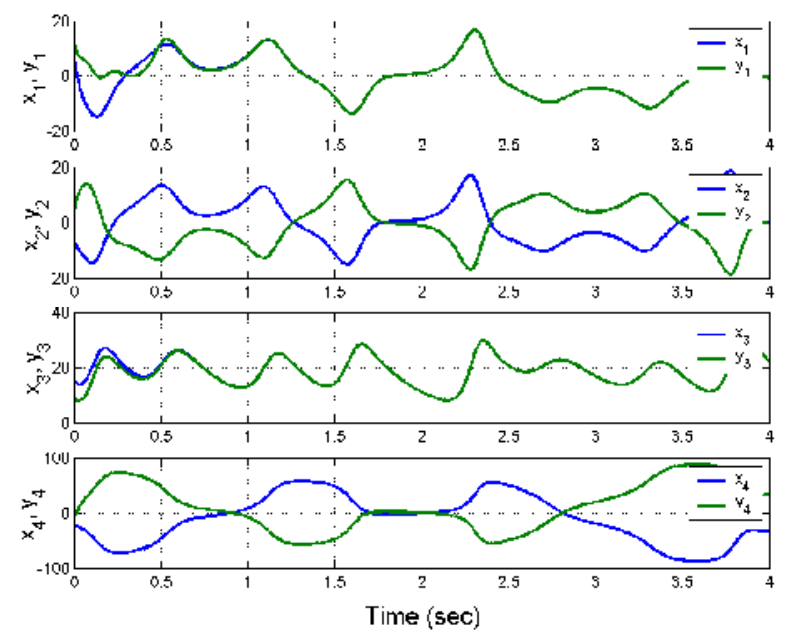

Figure 6. Hybrid Synchronization of Identical Hyperchaotic Li Systems 


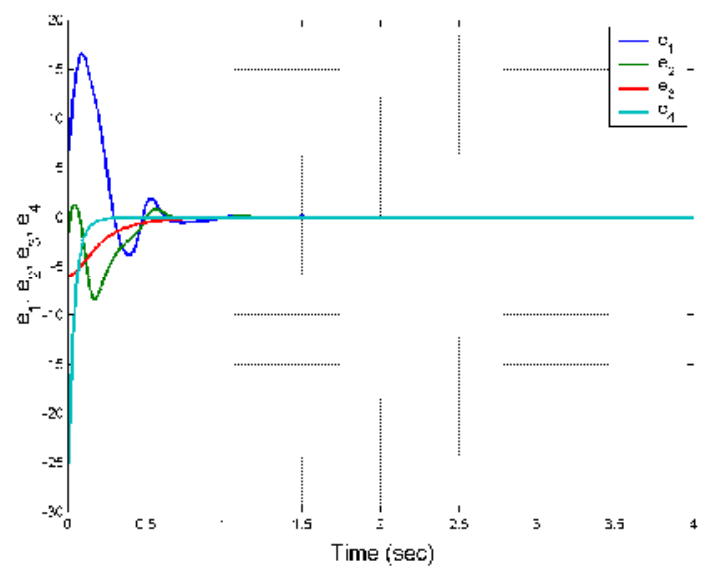

Figure 7. Time-History of the Hybrid Synchronization Errors $e_{1}, e_{2}, e_{3}, e_{4}$

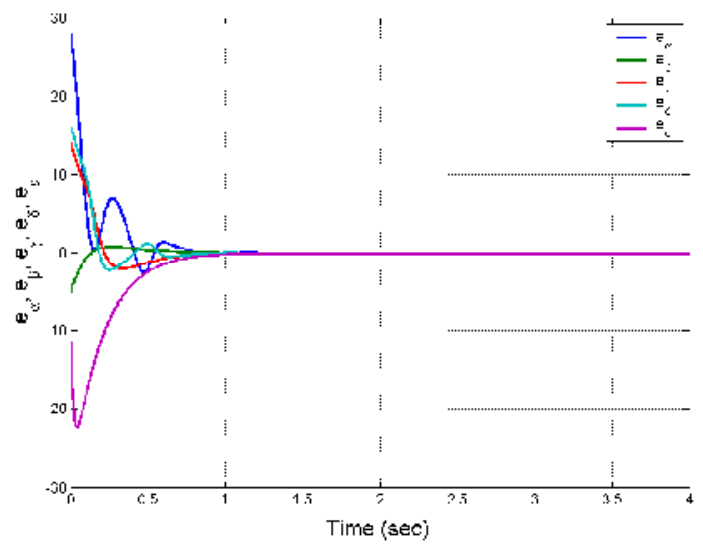

Figure 8. Time-History of the Parameter Estimation Errors $e_{\alpha}, e_{\beta}, e_{\gamma}, e_{\delta}, e_{\varepsilon}$

\section{Adaptive Controller Design for the Hybrid Synchronization DESIGN OF HYPERCHAOTIC XU AND HYPERCHAOTIC LI SYSTEMS}

In this section, we design an adaptive controller for the hybrid synchronization of hyperchaotic Xu system (2009) and hyperchaotic Li system (2005) with unknown parameters.

The hyperchaotic Xu system is taken as the master system, whose dynamics is given by

$$
\begin{aligned}
& \dot{x}_{1}=a\left(x_{2}-x_{1}\right)+x_{4} \\
& \dot{x}_{2}=b x_{1}+r x_{1} x_{3} \\
& \dot{x}_{3}=-c x_{3}-x_{1} x_{2} \\
& \dot{x}_{4}=x_{1} x_{3}-d x_{2}
\end{aligned}
$$

where $a, b, c, d, r$ are unknown parameters of the system.

The hyperchaotic Li system is also taken as the slave system, whose dynamics is given by 


$$
\begin{aligned}
& \dot{y}_{1}=\alpha\left(y_{2}-y_{1}\right)+y_{4}+u_{1} \\
& \dot{y}_{2}=\delta y_{1}-y_{1} y_{3}+\gamma y_{2}+u_{2} \\
& \dot{y}_{3}=-\beta y_{3}+y_{1} y_{2}+u_{3} \\
& \dot{y}_{4}=y_{2} y_{3}+\varepsilon y_{4}+u_{4}
\end{aligned}
$$

where $\alpha, \beta, \gamma, \delta, \varepsilon$ are unknown parameters and $u_{1}, u_{2}, u_{3}, u_{4}$ are the adaptive controllers. For the hybrid synchronization, the error $e$ is defined as

$$
e_{1}=y_{1}-x_{1}, e_{2}=y_{2}+x_{2}, e_{3}=y_{3}-x_{3}, e_{4}=y_{4}+x_{4}
$$

A simple calculation gives the error dynamics

$$
\begin{aligned}
& \dot{e}_{1}=\alpha\left(y_{2}-y_{1}\right)-a\left(x_{2}-x_{1}\right)+y_{4}-x_{4}+u_{1} \\
& \dot{e}_{2}=\delta y_{1}+\gamma y_{2}+b x_{1}+r x_{1} x_{3}-y_{1} y_{3}+u_{2} \\
& \dot{e}_{3}=-\beta y_{3}+c x_{3}+y_{1} y_{2}+x_{1} x_{2}+u_{3} \\
& \dot{e}_{4}=\varepsilon y_{4}-d x_{2}+y_{2} y_{3}+x_{2} x_{3}+u_{4}
\end{aligned}
$$

Next, we choose a nonlinear controller for achieving hybrid synchronization as

$$
\begin{aligned}
& u_{1}=-\hat{\alpha}(t)\left(y_{2}-y_{1}\right)+\hat{a}(t)\left(x_{2}-x_{1}\right)-y_{4}+x_{4}-k_{1} e_{1} \\
& u_{2}=-\hat{\delta}(t) y_{1}-\hat{\gamma}(t) y_{2}-\hat{b}(t) x_{1}-\hat{r}(t) x_{1} x_{3}+y_{1} y_{3}-k_{2} e_{2} \\
& u_{3}=\hat{\beta}(t) y_{3}-\hat{c}(t) x_{3}-y_{1} y_{2}-x_{1} x_{2}-k_{3} e_{3} \\
& u_{4}=-\hat{\varepsilon}(t) y_{4}+\hat{d}(t) x_{2}-y_{2} y_{3}-x_{2} x_{3}-k_{4} e_{4}
\end{aligned}
$$

where $k_{i},(i=1,2,3,4)$ are positive gains.

By the substitution of (44) into (43), the error dynamics is simplified as

$$
\begin{aligned}
& \dot{e}_{1}=(\alpha-\hat{\alpha}(t))\left(y_{2}-y_{1}\right)-(a-\hat{a}(t))\left(x_{2}-x_{1}\right)-k_{1} e_{1} \\
& \dot{e}_{2}=(\delta-\hat{\delta}(t)) y_{1}+(\gamma-\hat{\gamma}(t)) y_{2}+(b-\hat{b}(t)) x_{1}+(r-\hat{r}(t)) x_{1} x_{3}-k_{2} e_{2} \\
& \dot{e}_{3}=-(\beta-\hat{\beta}(t)) y_{3}+(c-\hat{c}(t)) x_{3}-k_{3} e_{3} \\
& \dot{e}_{4}=(\varepsilon-\hat{\varepsilon}(t)) y_{4}-(d-\hat{d}(t)) x_{2}-k_{4} e_{4}
\end{aligned}
$$

Next, we define the parameter estimation errors as

$$
\begin{aligned}
& e_{a}(t)=a-\hat{a}(t), \quad e_{b}(t)=b-\hat{b}(t), \quad e_{c}(t)=c-\hat{c}(t), e_{d}(t)=d-\hat{d}(t) \\
& e_{r}(t)=r-\hat{r}(t), \quad e_{\alpha}(t)=\alpha-\hat{\alpha}(t), e_{\beta}(t)=\beta-\hat{\beta}(t), e_{\gamma}(t)=\gamma-\hat{\gamma}(t) \\
& e_{\delta}(t)=\delta-\hat{\delta}(t), e_{\varepsilon}(t)=\varepsilon-\hat{\varepsilon}(t)
\end{aligned}
$$

Differentiating (46) with respect to $t$, we get 
International Journal of Advanced Information Technology (IJAIT) Vol. 3, No.2, April2013

$$
\begin{aligned}
& \dot{e}_{a}(t)=-\dot{\hat{a}}(t), \dot{e}_{b}(t)=-\dot{\hat{b}}(t), \dot{e}_{c}(t)=-\dot{\hat{c}}(t), \dot{e}_{d}(t)=-\dot{\hat{d}}(t), \dot{e}_{r}(t)=-\dot{\hat{r}}(t) \\
& \dot{e}_{\alpha}(t)=-\dot{\hat{\alpha}}(t), \dot{e}_{\beta}(t)=-\dot{\hat{\beta}}(t), \dot{e}_{\gamma}(t)=-\dot{\hat{\gamma}}(t), \dot{e}_{\delta}(t)=-\dot{\hat{\delta}}(t), \dot{e}_{\varepsilon}(t)=-\dot{\hat{\varepsilon}}(t)
\end{aligned}
$$

In view of (46), we can simplify the error dynamics (45) as

$$
\begin{aligned}
& \dot{e}_{1}=e_{\alpha}\left(y_{2}-y_{1}\right)-e_{a}\left(x_{2}-x_{1}\right)-k_{1} e_{1} \\
& \dot{e}_{2}=e_{\delta} y_{1}+e_{\gamma} y_{2}+e_{b} x_{1}+e_{r} x_{1} x_{3}-k_{2} e_{2} \\
& \dot{e}_{3}=-e_{\beta} y_{3}+e_{c} x_{3}-k_{3} e_{3} \\
& \dot{e}_{4}=e_{\varepsilon} y_{4}-e_{d} x_{2}-k_{4} e_{4}
\end{aligned}
$$

We take the quadratic Lyapunov function

$$
V=\frac{1}{2}\left(e_{1}^{2}+e_{2}^{2}+e_{3}^{2}+e_{4}^{2}+e_{a}^{2}+e_{b}^{2}+e_{c}^{2}+e_{d}^{2}+e_{r}^{2}+e_{\alpha}^{2}+e_{\beta}^{2}+e_{\gamma}^{2}+e_{\delta}^{2}+e_{\varepsilon}^{2}\right)
$$

which is a positive definite function on $R^{14}$.

When we differentiate (48) along the trajectories of (45) and (46), we get

$$
\begin{aligned}
\dot{V}= & -k_{1} e_{1}^{2}-k_{2} e_{2}^{2}-k_{3} e_{3}^{2}-k_{4} e_{4}^{2}+e_{a}\left[-e_{1}\left(x_{2}-x_{1}\right)-\dot{\hat{a}}\right]+e_{b}\left[e_{2} x_{1}-\dot{\hat{b}}\right]+e_{c}\left[e_{3} x_{3}-\dot{\hat{c}}\right] \\
& +e_{d}\left[-e_{4} x_{2}-\dot{\hat{d}}\right]+e_{r}\left[e_{2} x_{1} x_{3}-\dot{\hat{r}}\right]+e_{\alpha}\left[e_{1}\left(y_{2}-y_{1}\right)-\dot{\hat{\alpha}}\right]+e_{\beta}\left[-e_{3} y_{3}-\dot{\hat{\beta}}\right] \\
& +e_{\gamma}\left[e_{2} y_{2}-\dot{\hat{\gamma}}\right]+e_{\delta}\left[e_{2} y_{1}-\dot{\hat{\delta}}\right]+e_{\varepsilon}\left[e_{4} y_{4}-\dot{\hat{\varepsilon}}\right]
\end{aligned}
$$

In view of Eq. (50), we take the parameter update law as

$$
\begin{array}{lll}
\dot{\hat{a}}=-e_{1}\left(x_{2}-x_{1}\right)+k_{5} e_{a}, & \dot{\hat{b}}=e_{2} x_{1}+k_{6} e_{b}, & \dot{\hat{c}}=e_{3} x_{3}+k_{7} e_{c}, \\
\dot{\hat{d}}=-e_{4} x_{2}+k_{8} e_{d}, & \dot{\hat{r}}=e_{2} x_{1} x_{3}+k_{9} e_{r}, & \dot{\hat{\alpha}}=e_{1}\left(y_{2}-y_{1}\right)+k_{10} e_{\alpha}, \\
\dot{\hat{\beta}}=-e_{3} y_{3}+k_{11} e_{\beta}, & \dot{\hat{\gamma}}=e_{2} y_{2}+k_{12} e_{\gamma}, & \dot{\hat{\delta}}=e_{2} y_{1}+k_{13} e_{\delta}, \\
\dot{\hat{\varepsilon}}=e_{4} y_{4}+k_{14} e_{\varepsilon} &
\end{array}
$$

Theorem 6.1 The adaptive control law (44) along with the parameter update law (51), where $k_{i},(i=1,2, \ldots, 14)$ are positive gains, achieves global and exponential hybrid synchronization of the hyperchaotic Xu system (40) hyperchaotic Li system (41), where $\hat{a}(t), \hat{b}(t), \hat{c}(t), \hat{d}(t), \hat{r}(t)$, $\hat{\alpha}(t), \hat{\beta}(t), \hat{\gamma}(t), \hat{\delta}(t), \hat{\varepsilon}(t)$ are estimates of the unknown parameters $a, b, c, d, r, \alpha, \beta, \gamma, \delta, \varepsilon$, respectively. Moreover, all the parameter estimation errors converge to zero exponentially for all initial conditions.

Proof. We prove the above result using Lyapunov stability theory [25].

Substituting the parameter update law (51) into (50), we get 
International Journal of Advanced Information Technology (IJAIT) Vol. 3, No.2, April2013

$$
\begin{aligned}
\dot{V}= & -k_{1} e_{1}^{2}-k_{2} e_{2}^{2}-k_{3} e_{3}^{2}-k_{4} e_{4}^{2}-k_{5} e_{a}^{2}-k_{6} e_{b}^{2}-k_{7} e_{c}^{2}-k_{8} e_{d}^{2}-k_{9} e_{r}^{2} \\
& -k_{10} e_{\alpha}^{2}-k_{11} e_{\beta}^{2}-k_{12} e_{\gamma}^{2}-k_{13} e_{\delta}^{2}-k_{14} e_{\varepsilon}^{2}
\end{aligned}
$$

which is a negative definite function on $R^{14}$.

This shows that the hybrid synchronization errors $e_{1}(t), e_{2}(t), e_{3}(t), e_{4}(t)$ and the parameter estimation errors $e_{a}(t), e_{b}(t), e_{c}(t), e_{d}(t), e_{r}(t), e_{\alpha}(t), e_{\beta}(t), e_{\gamma}(t), e_{\delta}(t), e_{\varepsilon}(t)$ are globally exponentially stable for all initial conditions. This completes the proof.

Next, we demonstrate our hybrid synchronization results with MATLAB simulations.

The classical fourth order Runge-Kutta method with time-step $h=10^{-8}$ has been applied to solve the hyperchaotic Li systems (27) and (28) with the adaptive nonlinear controller (31) and the parameter update law (38). The feedback gains are taken as $k_{i}=5,(i=1,2, \ldots, 9)$.

The parameters of the hyperchaotic $\mathrm{Xu}$ and $\mathrm{Li}$ systems are taken as in the hyperchaotic case, i.e.

$$
a=10, b=40, c=2.5, r=16, d=2, \alpha=35, \beta=3, \gamma=12, \delta=7, \varepsilon=0.58
$$

For simulations, the initial conditions of the hyperchaotic Xu system (27) are chosen as

$$
x_{1}(0)=12, \quad x_{2}(0)=-4, \quad x_{3}(0)=8, \quad x_{4}(0)=-20
$$

Also, the initial conditions of the hyperchaotic Li system (28) are chosen as

$$
y_{1}(0)=21, \quad y_{2}(0)=7, \quad y_{3}(0)=19, \quad y_{4}(0)=-12
$$

Also, the initial conditions of the parameter estimates are chosen as

$$
\begin{aligned}
& \hat{a}(0)=-7, \quad \hat{b}(0)=14, \quad \hat{c}(0)=12, \quad \hat{d}(0)=-8, \quad \hat{r}(0)=15 \\
& \hat{\alpha}(0)=12, \quad \hat{\beta}(0)=5, \quad \hat{\gamma}(0)=4, \quad \hat{\delta}(0)=11, \quad \hat{\varepsilon}(0)=-6
\end{aligned}
$$

Figure 9 depicts the hybrid synchronization of hyperchaotic $\mathrm{Xu}$ and hyperchaotic Li systems.

Figure 10 depicts the time-history of the hybrid synchronization errors $e_{1}, e_{2}, e_{3}, e_{4}$.

Figure 11 depicts the time-history of the parameter estimation errors $e_{a}, e_{b}, e_{c}, e_{d}$.

Figure 12 depicts the time-history of the parameter estimation errors $e_{\alpha}, e_{\beta}, e_{\gamma}, e_{\delta}, e_{\varepsilon}$. 
International Journal of Advanced Information Technology (IJAIT) Vol. 3, No.2, April2013

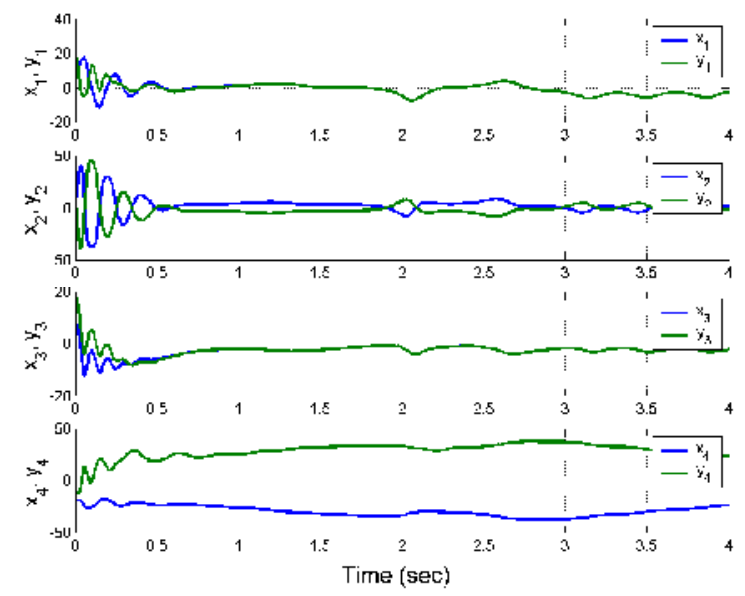

Figure 9. Hybrid Synchronization of Hyperchaotic Xu and Lu Systems

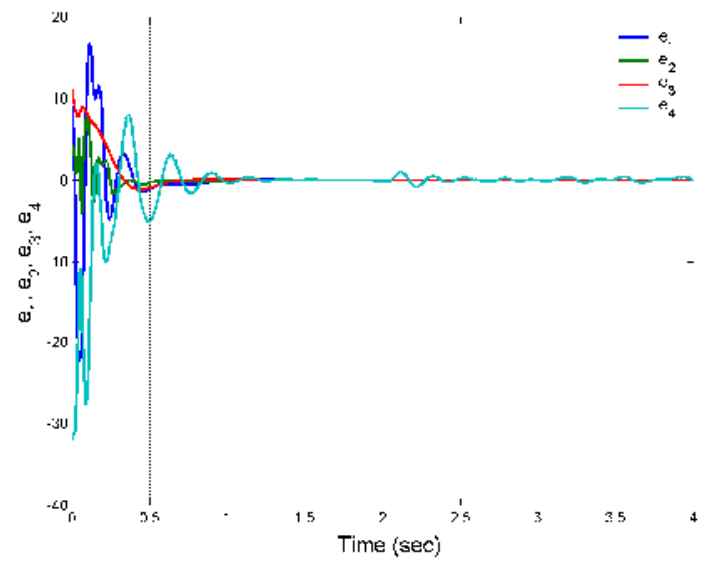

Figure 10. Time-History of the Hybrid Synchronization Errors $e_{1}, e_{2}, e_{3}, e_{4}$

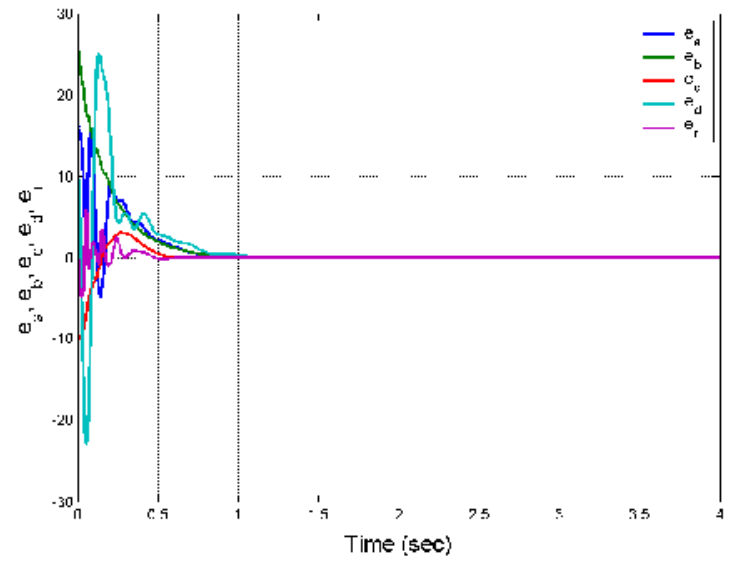

Figure 11. Time-History of the Parameter Estimation Errors $e_{a}, e_{b}, e_{c}, e_{d}$ 


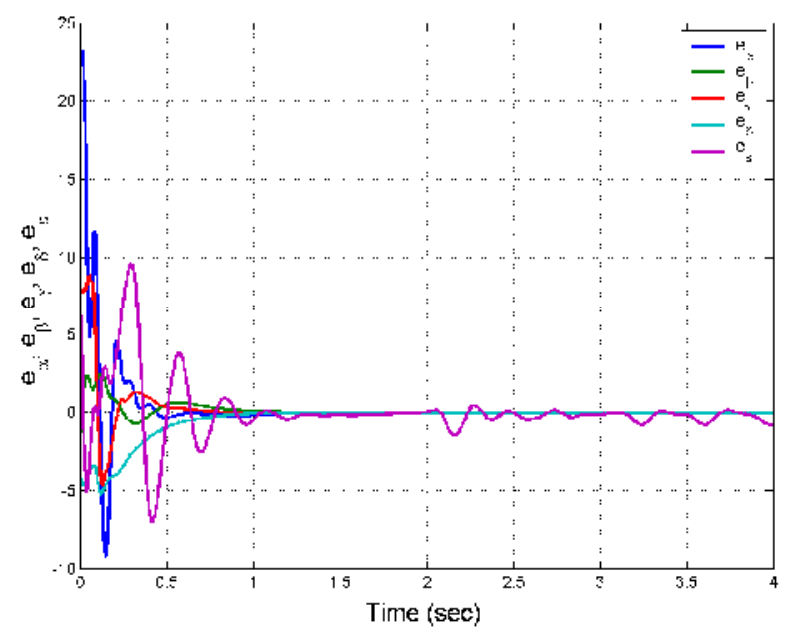

Figure 12. Time-History of the Parameter Estimation Errors $e_{\alpha}, e_{\beta}, e_{\gamma}, e_{\delta}, e_{\varepsilon}$

\section{Conclusions}

In this paper, using adaptive control method, we derived new results for the adaptive controller design for the hybrid synchronization of hyperchaotic Xu systems (2009) and hyperchaotic Li systems (2005) with unknown parameters. Using Lyapunov control theory, adaptive control laws were derived for globally hybrid synchronizing the states of identical hyperchaotic Xu systems, identical hyperchaotic Li systems and non-identical hyperchaotic Xu and Li systems. MATLAB simulations were displayed in detail to demonstrate the adaptive hybrid synchronization results derived in this paper for hyperchaotic Xu and Li systems.

\section{REFERENCES}

[1] Rössler, O.E. (1979) “An equation for hyperchaos,” Physics Letters A, Vol. 71, pp 155-157.

[2] Huang, Y. \& Yang, X.S. (2006) "Hyperchaos and bifurcation in a new class of four-dimensional Hopfield neural networks," Neurocomputing, Vol. 69, pp 13-15.

[3] Machado, L.G., Savi, M.A. \& Pacheco, P.M.C.L. (2003) "Nonlinear dynamics and chaos in coupled shape memory oscillators," International Journal of Solids and Structures, Vol. 40, No. 19, pp. 51395156.

[4] Tao, Y. (1999) "Chaotic secure communication systems - history and new results", Telecommun. Review, Vol. 9, pp 597-634.

[5] Li, C., Liao, X. \& Wong, K.W. (2005) "Lag synchronization of hyperchaos with applications to secure communications," Chaos, Solitons \& Fractals, Vol. 23, No. 1, pp 183-193.

[6] Prokhorov, M.D. \& Ponomarenko, V.I. (2008) "Encryption and decryption of information in chaotic communication systems governed by delay-differential equations," Chaos, Solitons \& Fractals, Vol. 35, No. 5, pp 871-877.

[7] Yassen, M.T. (2008) "Synchronization hyperchaos of hyperchaotic systems", Chaos, Solitons and Fractals, Vol. 37, pp 465-475.

[8] Ott, E., Grebogi, C. \& Yorke, J.A. (1990) “Controlling chaos”, Phys. Rev. Lett., Vol. 64, pp 11961199.

[9] Pecora, L.M. \& Carroll, T.L. (1990) "Synchronization in chaotic systems”, Phys. Rev. Lett., Vol. 64, pp 821-824.

[10] Bowong, S. \& Kakmeni, F.M.M. (2004) "Synchronization of uncertain chaotic systems via backstepping approach,” Chaos, Solitons \& Fractals, Vol. 21, No. 4, pp 999-1011.

[11] Suresh, R, \& Sundarapandian, V. (2012) "Global chaos synchronization of WINDMI and Coullet chaotic systems by backstepping control”, Far East J. Math. Sciences, Vol. 67, No. 2, pp 265-287. 
International Journal of Advanced Information Technology (IJAIT) Vol. 3, No.2, April2013

[12] Suresh, R. \& Sundarapandian, V. (2012) “Hybrid synchronization of n-scroll Chua and Lur'e chaotic systems via backstepping control with novel feedback", Arch. Control Sciences, Vol. 22, No. 3, pp 255-278.

[13] Senejohnny, D.M. \& Delavari, H. (2012) "Active sliding observer scheme based fractional chaos synchronization,” Comm. Nonlinear Sci. Numerical Simulation, Vol. 17, No. 11, pp 4373-4383.

[14] Sundarapandian, V. (2012) "Anti-synchronization of hyperchaotic Xu systems via sliding mode control", International Journal of Embedded Systems, Vol. 2, No. 2, pp 51-61.

[15] Sundarapandian, V. (2013) "Anti-synchronizing sliding controller design for identical Pan systems," International Journal of Computational Science and Information Technology, Vol. 1, No. 1, pp 1-9.

[16] Huang, L. Feng, R. \& Wang, M. (2004) "Synchronization of chaotic systems via nonlinear control," Physics Letters A, Vol. 320, No. 4, pp 271-275.

[17] Lei, Y., Xu, W. \& Zheng, H. (2005) "Synchronization of two chaotic nonlinear gyros using active control," Physics Letters A, Vol. 343, pp 153-158.

[18] Sundarapandian, V. (2012) "Adaptive control and synchronization of a generalized Lotka-Volterra system,” International Journal of Bioinformatics \& Biosciences, Vol. 1, No. 1, pp 1-12.

[19] Sundarapandian, V. (2013) "Adaptive controller and synchronizer design for hyperchaotic Zhou system with unknown parameters," International Journal of Information Technology, Modeling and Computing, Vol. 1, No. 1, pp 18-32.

[20] Zhao, J. \& Lü, J. (2006) "Using sampled-data feedback control and linear feedback synchronization in a new hyperchaotic system," Chaos, Solitons \& Fractals, Vol. 35, pp. 376-382.

[21] Ma, H., Deshmukh, V., Butcher, E. \& Averina, V. (2005) "Delayed state feedback and chaos control for time-periodic systems via a symbolic approach", Communications in Nonlinear Science and Numerical Simulation, Vol. 10, No. 5, pp 479-497.

[22] Xu, J., Cai, G. \& Zheng, S. (2009) "A novel hyperchaotic system and its control", Journal of Uncertain Systems, Vol. 3, No. 2, pp 137-144.

[23] Li, Y., Tang, W.K.S. \& Chen, G. (2005) "Generating hyperchaos via state feedback control", International Journal of Bifurcation and Chaos, Vol. 15, No. 10, pp 3367-3375.

[24] Sastry, S. \& Bodson, M. (2011) Adaptive Control: Stability, Convergence and Robustness, Dover.

[25] Hahn, W. (1967) The Stability of Motion, Springer, Berlin. 